Artículo de revisión

\title{
Estimulación de la conciencia a través de la meditación
}

\section{Consciousness stimulation by meditation}

\author{
Eliana Quintero-Gallego ${ }^{1 *}$, Angélica Flores ${ }^{2}$
}

1 Servicio de Neuropsicología, Unidad de Salud Mental, Instituto de Ortopedia Infantil Roosevelt. Bogotá, Colombia. 2 Departamento de Psicología Experimental, Universidad de Sevilla. Sevilla, España.

\section{Resumen}

El presente artículo presenta una relación entre la conciencia y meditación. Las teorías neurobiológicas y neurodinámicas han aportado datos empíricos y proponen métodos para estudiar la conciencia como un proceso cognitivo. Dentro de estas teorías se encuentran: la hipótesis del núcleo dinámico, la teoría del espacio global y el ciclo percepción-acción. En este artículo se partirá, en primer lugar, de una definición de conciencia desde una perspectiva neurocientífica y, a partir de allí, se abordará la meditación como estrategia para su estudio y estimulación. De las diversas técnicas de meditación nos centraremos en el mindfulness, por el impacto y acogida que ha tenido en la psicología.

Palabras clave: conciencia, meditación, conciencia plena

\section{Abstract}

This paper presents a relationship between consciousness and meditation. Neurobiological and neurodynamic theories have provided empirical data and propose methods to study consciousness as a cognitive process. Some of these theories are the dynamic core hypothesis, the theory of global space and perception-action cycle. This article will start with a definition of consciousness from a neuroscientific perspective, and from there, will address meditation as strategy to study and stimulate this process. Considering different techniques of meditation we focus on mindfulness meditation (MM) because its impact on psychology.

Keywords: consciousness, meditation, mindfulness

\footnotetext{
* Correspondencia: eliana_quintero@yahoo.es. Unidad de Salud Mental, Instituto de Ortopedia Infantil Roosevelt, Carrera 4 Este \#17-50, Bogotá D.C. Teléfono: +57 (1) 3534000

Recibido: 25-11-11. Revisión desde: 27-11-11. Aceptado: 17-01-12
}

DOI: $10.5839 / \mathrm{rcnp} .2012 .0701 .08$ 


\section{Introducción}

La conciencia ha sido estudiada desde aproximaciones filosóficas, psicológicas y actualmente, con las neurociencias han surgido teorías que aportan datos empíricos para explicar sus propiedades y correlatos cerebrales.

La filosofía occidental ha cuestionado la existencia física de la conciencia, la facultad del hombre para captar su propio pensamiento $\mathrm{y}$ ha planteado diferentes teorías explicativas (Searle, 2000). También los primeros psicólogos experimentales, como W. Wundt, intentaron comprender cómo se forman las sensaciones, las imágenes en el cerebro, la memoria, las percepciones de tiempo y espacio, y cuáles son los mecanismos que permiten que el individuo sea consciente de la información que está procesando (Rieber \& Robinson, 2001).

Las teorías neurobiológicas y neurodinámicas aportan datos y proponen métodos para estudiar la conciencia. Dentro de estas teorías están la "Hipótesis del núcleo dinámico" (Tononi \& Edelman, 1998; Edelman \& Tononi, 2000; Edelman, 2003; Tononi, 2001, 2004, 2008, 2010), la "Teoría del espacio global" (Baars, 1988; Baars et al., 2003) y el "Ciclo percepciónacción" (Perlovsky, 2006, 2009).

Una de las teorías más radicales y controvertidas es la Hipótesis del Núcleo Dinámico (Dynamic Core Hypotesis) (Edelman, 2003; Tononi \& Edelman, 1998). Sostiene que la conciencia es consecuencia de un proceso de interacción entre poblaciones neuronalesque se integran en la escala de milisegundos creando un complejo proceso neural unificado y diferenciado, generando la sensación consciente vinculada con una organización dinámica del sistema. Esta teoría asume que la conciencia no se atribuye a un conjunto reducido de neuronas y que el núcleo dinámico de la conciencia está cambiando su configuración neuronal constantemente y afecta las reacciones futuras por la influencia de los eventos externos e internos del organismo. También sostiene que la actividad consciente depende de la interacción entre las áreas tálamocortical y córticocorticales, y que cuando se interrumpe la interacción entre estas áreas se observa pérdida de conciencia (Holtzman \& Gazzaniga, 1985; Gazzaniga, 1995). Por ejemplo, cuando se presenta el síndrome de desconexión no hay una buena integración de la información y hay una disminución del estado de conciencia (Sperry, 1984). La actividad cortical de diferentes zonas se ha puesto de manifiesto a través del análisis de sincronía, en el que se analiza la coordinación del disparo neural en zonas locales o áreas generales, creando la coherencia y la unidad en el sistema (Edelman, 2003; Tononi, 2004). El núcleo dinámico surge a partir de la dinámica previa de la actividad sensomotora y la información contextual, se coordina espontánea y continuamente entre sí y se da por la integración local y segregación masiva de la información proveniente de las áreas cerebrales (Tononi \& Edelman, 1998; Edelman \& Tononi 2000; Tononi, 2001, 2004, 2010), generando un sistema complejo con funciones globales de alta coherencia que se manifiesta con una experiencia consciente (Edelman, 2003). Un estudio con tomografía por emisión de positrones (PET) en sujetos esquizofrénicos indica que el núcleo dinámico se encuentra reducido por la dificultad de integración global, lo cual se manifiesta como una alteración de su actividad consciente (Tononi, 1996).

Otro de los planeamientos explicativos de la conciencia humana es la Teoría del Espacio Global de Baars (Baars' Global Workspace Theory) (Baars, 1998, 2002; Baars et al., 2003). Esta teoría indica que la percepción consciente se genera por la integración de funciones inconscientes y sus componentes cerebrales, los cuales integran un espacio de trabajo, y por otro lado, la experiencia no consciente se da en áreas cerebrales separadas que procesan información específica. La teoría sugiere que el espacio de trabajo global coordina la interacción de los múltiples procesos descentralizados e inconscientes dando lugar a un mensaje global que será transmitido al sistema total. Esta teoría ha sido revisada y comprobada con estudios de EEG, neuroimagen e investigación computacional (Dehaene et al., 2001; Gaillard et al., 2009). Además, plantea que para que se presente un estado consciente debe haber una coordinación temporal en el disparo neural el cual es limitado en el tiempo (Cleeremans \& Sarrazin, 2007).

Por otro lado, Perlovsky $(2006,2009)$ plantea el Ciclo percepción-acción (action-perception-cicle) para explicar la conciencia. Este ciclo sugiere que el cerebro es un sistema complejo que va de un estado de altas dimensiones caóticas, en el que está presente lo vago, nuboso e inconsciente, a un estado de bajas dimensiones caóticas, el cual es concreto, conciso y consciente. También indica que el estado consciente permite la diferenciación para seleccionar objetivos específicos y concentrar la energía en los objetivos más importantes de cada momento, y cuando el individuo toma una decisión deliberada se genera una nueva situación con cambios ambientales que lo llevan nuevamente a un estado de altas dimensiones caóticas. Es decir, la conciencia se debe a la interacción entre los procesos bottom-up y top-down, como procesos de adaptación entre estados vagos y nítidos que llevarán al individuo a tomar decisiones, y estas a su vez generan cambios en su sistema dinámico y complejo. Los datos empíricos con EEG en animales y humanos apoyan este plan-teamiento al demostrar que la desincronización espacio-temporal del cerebro está relacionada con patrones de estados mentales con altas dimensiones caóticas, inespecíficos e impredecibles, mientras que la alta sincronización se relaciona con estados de bajas dimensiones caóticas (Freeman, 2007; Baijal, 2010).

\section{La meditación: estrategia para estimular la conciencia}

La meditación se originó como una práctica espiritual en diferentes partes del mundo (Sivananda, 1977; Patanjali, 2008). A partir de 1950 se han realizado estudios científicos sobre sus beneficios físicos y mentales y también sobre los cambios producidos en el estado de conciencia de los practicantes (Lutz et al., 2004; Slagter et al., 2007). La meditación es una técnica que busca reducir, controlar y tomar distancia de los pensamientos y/o sentimientos (Rubia, 2009) que llegan a ser una fuente de angustia, temor y preocupación (Jevining et al., 1992; Young \& Taylor, 2001; Cahn \& Polich, 2006; Walsh \& Shapiro, 2006; Brefczynski-Lewis et al., 2007; Raffune \& Srinvasan, 2009). Existe un amplio repertorio de técnicas de meditación; sin embargo, como propone Goleman (1976), "todas son variaciones de un proceso único para la transformación de la conciencia".

Las técnicas de meditación se dividen considerando la forma como se orientan los procesos atencionales (Lutz et al., 2008; Dakwar \& Levin, 2009; Raffune \& Srinvasan, 2009). En este sentido se encuentran:

1. Técnicas de atención focalizada, en las cuales la atención se centra y se sostiene de manera voluntaria sobre un 
objeto de meditación (por ejemplo, un mantra, una vela, una imagen, la respiración, una emoción, entre otras), y se caracterizan por un patrón electrofisiológico con ondas beta-2 (20-30 Hz) y gamma (30-50 Hz) (Cahn et al., 2010).

2. Técnicas de monitoreo abierto de la experiencia, que estimulan la observación de los pensamientos y sentimientos (sin juicios, reacciones ni identificaciones). Se caracterizan por predominio de las frecuencias theta frontal $(5-8 \mathrm{~Hz})$ y gama occipital (30-50 Hz) (Cahn et al., 2010). Dentro de este grupo se encuentra la "meditación mindfulness" (MM), cuyos conceptos básicos han sido utilizados en diversas técnicas y programas de intervención psicológica. Al respecto, Bishop y colaboradores (2004) sugieren que las habilidades vinculadas a MM han sido adaptadas como una aproximación terapeútica para "incrementar la conciencia y la capacidad de responder hábilmente a los procesos mentales que contribuyen al malestar emocional y a la conducta desadaptativa".

3. Además de estos dos grupos, Travis y Shear (2010) sugieren una tercera categoría dentro de la práctica de meditación que denominan: "automatic self-transcending" (Travis \& Shear, 2010). En este grupo se encuentran las técnicas de meditación trascendental, que se asocian con ritmos alfa 1 (7-9 $\mathrm{Hz}$ ), conducen automáticamente a la experiencia de conciencia $y$, a diferencia de las técnicas anteriores, no requieren control sobre el procesamiento cognitivo.

Algunos autores proponen un modelo neurobiológico de la meditación que incluye la activación de un sistema complejo de estructuras cerebrales y del sistema nervioso parasimpático $y$, adicionalmente, cambios neuroquímicos (Newberg \& Ivversen, 2003). Sin embargo, las estrategias de meditación son diferentes, asimismo los procesos cognitivos involucrados y la activación cerebral subyacente. De forma que, si en la meditación se focaliza la atención en un sentimiento de compasión, se produce un estado de coherencia en la banda gamma; mientras que si la meditación está orientada hacia la auto trascendencia (por ejemplo, meditación trascendental), habría mayor coherencia en el ritmo alfa (Travis, comunicación personal). En la $\mathrm{MM}$ aún no se han realizado estudios sobre coherencia cerebral; sin embargo, se ha descrito activación de la corteza prefrontal dorsolateral y de zonas mediales del córtex prefrontal (Simon, 2007).

Se han comparado los ritmos cerebrales producidos con técnicas de meditación focalizada y aquellos generados con técnicas de meditación abierta (como la MM y la meditación vipassanna). Dunn y colaboradores (1999) encontraron que la meditación abierta estaba asociada con una mayor actividad cerebral en las bandas de frecuencia delta, theta, alfa y beta. La actividad theta se localizó principalmente en los lóbulos frontales y la actividad delta, alfa y beta se extendió a través de los lóbulos frontales, temporales y parietales. Asimismo, Cahn y colaboradores (2010) informaron del patrón del EEG durante una meditación de monitoreo abierto (tipo MM). En su estudio midieron la actividad eléctrica cerebral a 16 individuos que habían practicado meditación durante aproximadamente 20 años. Durante la meditación se obtuvieron mayores ritmos theta $(4-8 \mathrm{~Hz}$ ) en el nivel frontal y ritmos gamma en el nivel occipital. En otro estudio, Lutz y colaboradores (2004) concluyeron que la evocación del estado de compasión (que también surge en la MM) generaba oscilaciones de gran amplitud en la banda de frecuencia gamma, especialmente en electrodos laterales fronto-parietales.
Aún no es del todo claro el papel cognitivo de dichas modificaciones electrofisiológicas. Se ha sugerido que el incremento en theta $(4 \mathrm{a} 8 \mathrm{~Hz}$ ) en regiones anteriores podría ser específico de la práctica de meditación (Aftanas \& Golocheikine, 2001, 2003), ser más notorio en los practicantes avanzados (Aftanas \& Golocheikine, 2001; Lehmann et al., 2001) y favorecer una sensación del calma y tranquilidad. Las bandas beta y alfa, generadas en regiones posteriores, podrían mantener al meditador alerta y consciente del entorno (Dunn et al., 1999). En cuanto a la banda de frecuencia gamma, se ha indicado que se asocia con el procesamiento significativo y la integración de los estímulos de manera coherente (Stern et al., 2001).

Los estudios con resonancia magnética funcional han demostrado cambios estructurales dados por engrosamiento cortical en varias regiones cerebrales (ínsula anterior derecha y corteza prefrontal derecha) en los meditadores con mayor experiencia (Lazar et al., 2005). Farb y colaboradores (2007) encontraron que los participantes entrenados en MM mostraban una mayor activación de áreas viscero-somáticas, incluida la ínsula derecha. De otro lado, Holzel y colaboradores (2008), utilizando morfometría de voxels, hallaron evidencia de que la práctica regular de la meditación está asociada con diferencias estructurales en regiones como la ínsula, el córtex órbito-frontal y el hipocampo.

Las investigaciones sobre la actividad cerebral relacionada con la meditación han concedido especial importancia a la corteza prefrontal (Simon, 2007). Esta estructura se relaciona con la integración y organización de la información proveniente de otras regiones cerebrales; también se encarga de las funciones ejecutivas y, finalmente, permite desarrollar niveles de conciencia (Stuss \& Benson, 1986). La conciencia plena (MM) sobre las sensaciones del cuerpo favorece la identificación de síntomas de malestar físico que son indicadores de posibles enfermedades. De igual forma, la conciencia en el nivel emocional, facilita la expresión de emociones de manera sana en diversas circunstancias de la vida. Esta autoregulación estimulada con la MM permite conservar un estado físico y mental saludable (Brown y Ryan, 2003; Shapiro y Schwartz, 2000). La MM favorece un procesamiento más consciente de la información, eliminando los automatismos a los cuales se está acostumbrado (Simon, 2007).

\section{Conclusiones}

Actualmente, un gran número de observaciones y experimentos contribuyen a la comprensión de la conciencia. La importancia de estudiar la conciencia y las técnicas que la estimulan radica en que es un proceso adaptativo que permite al organismo concentrar la energía en los objetivos que son más importantes en cada momento (Perlovsky, 2006, 2009).

Como se ha mencionado, la percepción consciente implica la integración de la información (Edelman \& Tononi, 1998; Edelman et al., 2011; Baars, 2002; Baars et al., 2003); también se ha sugerido que el contenido de la percepción consciente podría ser regulado por el individuo al dirigir su atención sensorial en un tema específico (Knudsen, 2007). En ese sentido, Sivananda (1977) indica que la atención es enfocar la conciencia sobre algún objeto elegido y si esta existe también habrá concentración. Por lo anterior, podemos sugerir que la meditación, al favorecer el control de la atención, también favorece la conciencia, es decir, que la regulación atencional podría estar 
favoreciendo la integración de información de un tema específico en un momento determinado y, consecuentemente, permitirá que el individuo tome decisiones adaptativas en el aquí y ahora.

Durante la experiencia consciente y en la meditación intervienen varias estructuras cerebrales. La teoría del núcleo dinámico (Edelman \& Tononi, 1998, 2000, 2008) ha sugerido que la conciencia está relacionada con redes cortico-talámicas y áreas corticales (Edelman \& Tononi, 1998, 2000, 2008). La activación de la corteza prefrontal es necesaria para generar una experiencia consciente, ya que interviene en la memoria y en la planificación (Edelman, 1989; Edelman et al., 2011). Sin embargo, es la interacción entre estructuras frontales y la corteza sensorial la que permite crear una percepción y experiencia consciente (Baars et al., 2003). En cuanto a las prácticas basadas en la meditación, se ha sugerido que involucran diversos componentes del sistema nervioso autónomo, estructuras cerebrales corticales y el tallo cerebral (Newberg \& Iversen, 2003). Se ha encontrado activación en áreas hipocampales y parahipocampales (asociadas con la memoria y aspectos de regulación emocional) y en regiones prefrontales y del giro cingular anterior (asociadas con procesos atencionales), y disminución de la actividad en los lóbulos parietales (asociados con el procesamiento espacial) (Newberg \& Iversen, 2003; Cahn \& Polich, 2006). Por lo tanto, sugerimos que la activación de algunas estructuras frontales durante la meditación podría, junto con la activación simultánea de otras estructuras del SNC, explicar la experiencia de "conciencia".

Los resultados experimentales también indican que durante una experiencia consciente mejora la coherencia en la frecuencia de la banda gamma (Engel \& Singer, 2001). Estos datos sugieren que las bases físicas de la conciencia están distribuidas en el cerebro y que es necesaria una coordinación espacio-temporal en diferentes grupos de neuronas (Baars, 1988; Edelman, 2003).También se ha relacionado la alta sincronización con estados de bajas dimensiones caóticas (Freeman, 2007; Baijal, 2010). Aunque aún no hay estudios sobre la coherencia en el EEG en MM, los estudios con meditación trascendental muestran que la experiencia de conciencia se correlaciona con el aumento de coherencia en el EEG (Levine et al., 1977) y esto se asocia con un mejor procesamiento de la información. Lutz y colaboradores (2004) encontraron un aumento en la coherencia en el rango de las frecuencias gamma $(25-42 \mathrm{~Hz})$. Estos datos sugieren que tanto la experiencia consciente como la meditación permiten la sincronización neuronal, lo que puede llevar a experimentar un estado claro, concreto, conciso y consciente.

La propuesta de Perlovsky hace referencia a dos estados dentro de un ciclo, uno en el que predomina lo vago, nuboso e inconsciente, y otro concreto, conciso y más consciente. Lo anterior se puede relacionar con el estado de "mindfulness" que se refiere a la conciencia de la experiencia y contrasta con un estado de menor conciencia y conductas de carácter automático denominado "mindlessness" (Brown y Ryan, 2003). El individuo que actúa de manera automática y en el que predominan los pensamientos y sentimientos del pasado y del futuro, toma decisiones inadecuadas, se aferra a esos pensamientos y no logra percibir falencias físicas y emocionales que constituyen señales de alarma para muchas patologías. En contraste, al "permanecer en el presente" se obtiene un estado de unidad e integridad $y$, por ende, un estado de conciencia organizada. Por lo tanto, la meditación permite la integración de la información y lleva a estados organizados y con bajos niveles de dimensiones caóticas.

Desde la neurociencia se han propuesto modelos para explicar la conciencia, sus propiedades y su referente neurobiológico; en tanto que el abordaje de la conciencia desde las técnicas de meditación ha sido más experiencial. A pesar de ello, las técnicas de meditación se han venido utilizando de manera efectiva y a bajo costo para abordar diferentes patologías psicológicas y médicas. Así mismo, los cambios neurológicos producidos en los meditadores expertos aportan mayor información sobre los correlatos electrofisiológicos de la conciencia (Lutz et al., 2004). Este artículo es una invitación a reflexionar y buscar respuestas sobre los planteamientos que sugieren cambios cerebrales durante y después de la meditación y cómo estos se correlacionan con la conciencia descrita desde las neurociencias.

\section{Referencias}

Aftanas, L. I. \& Golocheikine, S. A. (2001). Human anterior and frontal midline theta and lower alpha reflect emotionally positive state and internalized attention: High resolution EEG investigation of meditation. Neuroscience Letters, 310, 57-60.

Aftanas, L. I. \& Golocheikine, S. A. (2003). Changes in cortical activity in altered states of consciousness: The study of meditation by high-resolution EEG. Human Physiology, 29, 143-151.

Baars B. J. (1988). A Cognitive Theory of Consciousness. Cambridge: Cambridge University Press, 1988.

Baars, B. J. (2002). The conscious access hypothesis: Origins and recent evidence. Trends in Cognitive Science, 6, 47-52.

Baars, B. J., Ramsoy, T. Z. \& Laureys, S. (2003). Brain, conscious experience and the observing self. Trends in Neuroscience, 26, 671-675.

Baijal, S. \& Srinivasan, N. (2010). Theta activity and meditative states: spectral changes during concentrative meditation. Cognitive processing, 11(1), 31-38.

Bishop, S. R., Lau, M., Shapiro, S., Carlson, L., Anderson, N. D., Carmody, J., Segal, V. Z., Abbey, S., Speca, M., Velting, D. \& Devins, G. (2004). Mindfulness: a proposed operational definition. Clinical Psychology: Science and Practice, 11, 230-241.

Brefczynski-Lewis, J. A., Lutz, A., Schaefer, H. S., Levinson, D. B. \& Davidson, R. J. (2007). Neural correlates of attentional expertise in long-term meditation practitioners. Proc. Natl. Acad. Sci. U.S.A, 104, 11483-11488.

Brown, K. W. \& Ryan, R. (2004). Perils and promise in defining and measuring mindfulness: observations from experience. Clinical Psychology: Science and practice, 11, 242-248.

Cahn, B. R. \& Polich, J. (2006). Meditation states and traits: EEG, ERP, and neuroimaging studies. Psychological Bulletin, 132, 180-211.

Cahn, B. R., Delorme, A., \& Polich, J. (2010). Occipital gamma activation during Vipassana meditation. Cognitive Processing, 11(1), 39-56.

Cleeremans, A., \& Sarrazin, J. C. (2007). Time, action, and consciousness. Human Movement science, 26(2), 180-202.

Dakwar, E. \& Levin, F. (2009). The Emerging Role of Meditation in Addressing Psychiatric Illness, with a Focus on Substance Use Disorders. Harvard Review of Psychiatry, 17(4), 254-267.

Dehaene, S., Naccache, L., Cohen, L., Bihan, D., Mangin, J., Poline, B., et al. (2001). Cerebral mechanisms of word masking and unconscious repetition priming. Nature Neuroscience, 4, 752-758.

Dunn, B. R., Hartigan, J. A. \& Mikulas, W. L. (1999). Concentration and mindfulness meditations: Unique forms of consciousness? Applied Psychophysiology and Biofeedback, 24, 147-165.

Edelman, G. M., Tononi, G. A. (2000). Universe of consciousness. New York: Basic Books.

Edelman, G. M. (2003). Naturalizing consciousness: a theoretical framework. Proceedings of the National Academy of Sciences of the United States of America, 100, 5520-5524.

Edelman, G. M., Gally, J. A. \& Baars, B. J. (2011). Biology of consciousness. Frontiers in Psychology, 2, 4.

Engel, A. \& Singer, W. (2001). Temporal binding and the neural correlates of sensory awareness. Trends in Cognitive Sciences, 5, 16-25.

Farb, N. A. S., Segal, Z.V., Mayberg, H., et al. (2007). Attending to the present: meditation reveals distinct neural modes of self-reference. Social Cognitive and Affective Neuroscience, 2, 313-322. 
Freeman, W. J. (2001). Neurodynamics. An Exploration of Mesoscopic Brain Dynamics. London: Springer, 2001.

Freeman, W. J. (2007). Proposed Cortical "Shutter" Mechanism in Cinematographic Perception. Chapter 2. Perlovsky L. I. \& Kozma, R. Neurodynamics of Higher-Level Cognition and Consciousness. Heidelberg, Germany: SpringerVerlag.

Holtzman, D. \& Gazzaniga, M. S. (1985). Enhanced dual task performance following corpus commissurotomy in humans. Neuropsychologia, 23(3), 315-21.

Gazzaniga, M.S. (1995). Principles of human brain organization derived from splitbrain studies. Neuron, 14(2), 217-228.

Gaillard, R., Dehaene, S., Adam, C., Cle'menceau, S., Hasboun, D., et al. (2009). Converging intracranial markers of conscious access. PLoS Biology, 7(3), e61.

Goleman, D. (1976). Los caminos de la meditación. Barcelona: Kairos.

Holzel, B. K., Ott, U., Gard, T., Hempel, H., Weygandt, M., Morgen, K \& Vaitl, D. (2008). Investigation of mindfulness meditation practitioner with voxel-based morphometry. Scan, 3, 55-61.

Jevning, R., Wallace, R. K. \& Beidebach, M. (1992). The physiology of meditation a review - a wakeful hypometabolic integrated response. Neuroscience and Biobehavioral Reviews, 16(3), 415-424.

Knudsen, E. I. (2007). Fundamental components of attention. Annual Review of Neuroscience, 30, 57-78.

Lazar, S. W., Kerr, C. E., Wasserman, R. H., et al. (2005). Meditation experience is associated with increased cortical thickness. Neuroreport, 16, 1893-1897.

Lehmann, D., Faber, P. L., Achermann, P., Jeanmonod, D., Gianotti, L. R. R. \& Pizzagalli, D. (2001). Brain sources of EEG gamma frequency during volitionally meditation-induced, altered states of consciousness, and experience of the self. Psychiatry Research: Neuroimaging, 108, 111-121.

Lutz, A., Greischar, L. L., Rawlings, N. B., Ricard, M. \& Davidson, R. J. (2004). Longterm meditators self-induce high-amplitude gamma synchrony during mental practice. Proceedings of the National Academy of Sciences USA, 101, 1636916373.

Lutz, A., Slagter, H. A., Dunne, J. D. \& Davidson, R. J. (2008). Attention regulation and monitoring in meditation. Trends in Cognitive Sciences, 12, 163-169.

Newberg, A. B. \& Iversen, J. (2003). The neural basis of the complex mental task of meditation: Neurotransmitter and neurochemical considerations. Medical Hypotheses, 61, 282-291.

Patanjali (2008). Yoga Sutras. El sendero del yoga. Argentina: Editorial Hastinapura.

Raffone, A. \& Srinivasan, N. (2009). An adaptive workspace hypothesis about the neural correlates of consciousness: insights from neuroscience and meditation studies. Progress in Brain Research, 176, 161-180.

Perlovsky, I. L. (2006). Toward physics of the mind: Concepts, emotions, consciousness, and symbols. Physics of Life Reviews, 3, 23-55

Perlovsky, L. I. (2009). "Vague to crisp" neural mechanism of perception. IEEE transactions on neural networks, 20(8), 1363-1367.

Rubia, K. (2009). The neurobiology of Meditation and its clinical effectiveness in psychiatric disorders. Biological Psychology, 82, 1-11.

Rieber, W. R. \& Robinson K. D. (2001). Wilhelm Wundt in history: the making of a scientific psychology. New York : Kluwer Academic/Plenum.

Shapiro, S. L. \& Schwartz, G. E. (2000). The role of intention in self regulation: toward intentional systematic mindfulness. In M. Boekaerts, P.R. Pintrich \& M. Zeidener (Eds.) Handbook of self regulation. NY: Academic Press, 253-273.

Searle, R. J. (2000). El misterio de la conciencia. Barcelona: Paidós.

Simon, V. M. (2007). Minfulness \& neurobiologia. Revista de Psicoterapia, 66/67, 5-30.

Sivananda, S. (1977). Concentración y meditación. Sobre la energía interna del hombre y su aplicación en la vida diaria. Madrid: Gráficas Halar.

Sperry, R. (1984). Consciousness, personal identity and the divided brain. Neuropsychologia, 22(6), 661-673.

Stern, R. M., Ray, W. J. \& Quiglyey, K. S. (2001). Psychophysiological recording, $2^{\text {nd }}$ ed. New York: Oxford University Press.

Stuss, D. T. \& Benson, D. F. (1986). The frontal lobes. NY: Raven Press.

Tononi, G. \& Edelman, G. M. (1998). Consciousness and complexity. Science, 282, 1846-1851.

Tononi, G. (2001). Information measures for conscious experience. Archives Italiennes de Biologie,139, 367-371.

Tononi, G. (2004). An information integration theory of consciousness. BMC Neuroscience, 2(5), 42.

Tononi, G. (2008). Consciousness as integrated information: a provisional manifesto. Archives Italiennes de Biologie, 215, 216-242.

Tononi, G. (2010). Information integration: its relevance to brain function and consciousness. Archives Italiennes de Biologie, 148(3), 299-322.

Travis, F \& Shear, J. (2010). Focused attention, open monitoring and automatic self transcending: categories to organize meditations from vedic, Buddhist and Chinese traditions. Conscious Cogn,19(4), 1110-1118.

Walsh, R. \& Shapiro, S. L. (2006). The meeting of meditative disciplines and Western psychology: a mutually enriching dialogue. Am Psychol, 61, 227-239.

Young, J. D. \& Taylor, E. (2001). Meditation as a voluntary hypometabolic state of biological estivation. News in Physiological Sciences, 13, 149-153. 